\title{
RESEARCH HIGHLIGHT Females pay a higher price for addiction
}

\author{
Natalie E. Zlebnik (iD) \\ Neuropsychopharmacology (2019) 44:1179-1181; https://doi.org/10.1038/s41386-019-0373-0
}

Clinical evidence suggests that females are more vulnerable to drug addiction than males, transitioning from recreational use to abuse more rapidly and exhibiting a higher risk of relapse [1, 2]. Rodent models of addiction support these sex differences and indicate that females may be more susceptible to cocaine selfadministration and relapse than males due to biological factors, including cycling ovarian hormones and organizational differences in neurobiology. Among females, enhanced cocainemotivated behaviors are associated with phases of the menstrual (human) or estrous (rodent) cycle when circulating levels of estrogen are elevated, such as the follicular (human) or pro/estrus (rodent) phases (Fig. 1a). Compared to low estrogen levels, females with high circulating estrogen demonstrate greater cocaine craving and heightened subjective effects of cocaine. However, the ovarian hormone cycle may play a broader role in cocaine addiction by influencing the formation of associations between cocaine and environmental cues. Cues are theorized to acquire incentive motivational properties due to their association with drug delivery, and they can exert powerful control over drug seeking, often precipitating craving and relapse even after months of abstinence [3]. According to a recent rodent study [4], estrous cycle stage may play a crucial role in establishing Pavlovian associations among contextual cues and cocaine. Females in pro/estrus exhibit enhanced conditioned cocaine reward, forming stronger cocaine-cue associations compared to females in diestrus. These findings suggest that female vulnerability to addiction may extend to the influence of drug-paired stimuli and have significant consequences for craving and relapse prevention.

In this issue, Johnson et al. [5] examine whether estrous cycle stage at time of conditioning of cocaine cues influences the motivational impact of those cues on future cocaine selfadministration. Standard preclinical drug self-administration procedures often present cue lights and/or tones prior to or coincident with response-contingent i.v. drug infusions and thus preclude the consideration of cue learning in the absence of acute drug reinforcement. Here, Pavlovian conditioning is used to establish cocaine-cue associations independently of cocaine selfadministration (Fig. 1b). Then, using Pavlovian-to-instrumental transfer (PIT), the incentive motivational properties of the conditioned cue can be evaluated by measuring its ability to invigorate or potentiate instrumental responding for cocaine (Fig. 1c). As stage-specific experimental testing is inherently constrained by the 4-day estrous cycle, a threshold selfadministration procedure was chosen that examines multiple doses of cocaine and provides independent assessment of both consummatory and motivational aspects of cocaine selfadministration within a single session. Principles of behavioral economics can be applied to determine preferred cocaine consumption at low response costs $\left(Q_{0}\right)$ and also the highest price paid to maintain stable cocaine intake $\left(P_{\text {max }}\right)$.

Results reveal a critical role for cues conditioned in pro/estrus in enhancing motivation for cocaine. No sex-dependent or cycledependent differences in cocaine self-administration were demonstrated in the absence of cues, and when presented noncontingently during the threshold cocaine self-administration session, conditioned cues did not affect $Q_{0}$ or cocaine consumption under unrestrained conditions. However, cues previously paired with non-contigent i.v. cocaine infusions during pro/estrus -but not in diestrus or in males-potently elevated $P_{\max }$ or maximal price paid compared to sessions without the cue present (Fig. 1c). Therefore, females presented with pro/estrus-conditioned cues exert greater effort or pay a much higher price for each milligram of cocaine compared to control conditions. Importantly, this incentive motivational effect of the pro/estrus-conditioned cue was not dependent on current cycle stage, revealing the broad impact of phase-specific conditioning on future motivated behavior.

Lack of sex-dependent and estrous cycle-dependent differences in cocaine self-administration in the absence of discrete cues is noteworthy. This suggests that previous sex differences in cocaine self-administration may be largely driven by cocaine-paired cues. However, robust sex differences are generally revealed at low-tomoderate cocaine doses and under extended access conditions [6]. Therefore, the current study may not be optimized to confirm sex and/or estrous cycle effects shown previously. Rather, the conditions of the present study are well-suited to isolate the effects of cocaine-paired cues due to the absence of overt baseline sex and cycle differences. Future work may reveal interactions between pro/estrus-conditioned cocaine cues and current cycle status, but the present study contributes to our growing understanding of the complexity of cue-mediated factors that interact to promote and sustain sex differences in cocaine addiction.

Additional experiments demonstrate that cocaine-conditioned cues themselves support operant behavior and recruit brain regions associated with cocaine seeking. On Day 1 and Day 30 after cocaine self-administration training, conditioned cues were used to reinforce lever-pressing in the absence of cocaine (Fig. 1d). On Day 30, cues conditioned during pro/estrus maintained instrumental responding at greater levels than cues conditioned during diestrus or in males. Further, these same cues differentially activated striatal regions critical for motivated behavior. Specifically, responding for cues conditioned in pro/estrus compared to the other conditions elevated c-Fos expression in the dorsolateral striatum and nucleus accumbens shell and core, where c-Fos was positively correlated with cue seeking. Together, these findings underscore cycle-dependent differences in cue conditioning and

${ }^{1}$ Department of Anatomy and Neurobiology, University of Maryland School of Medicine, 20 Penn Street, Baltimore, MD 21201, USA

Correspondence: Natalie E. Zlebnik (nzlebnik@som.umaryland.edu)

Received: 12 March 2019 Accepted: 13 March 2019

Published online: 26 March 2019 


\section{A Human}

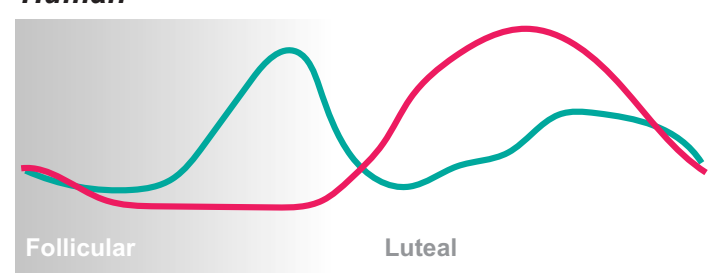

B

Pavlovian pairings

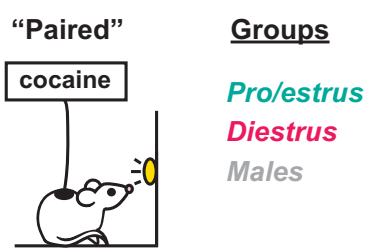

"Unpaired"

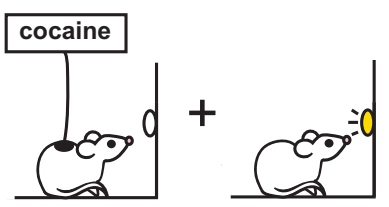

\section{C} Behavioral economics + PIT
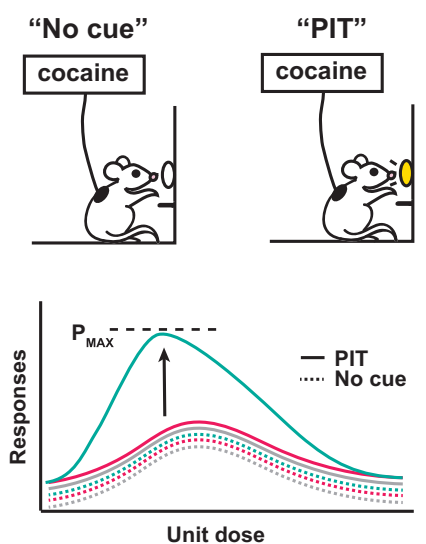

Rodent

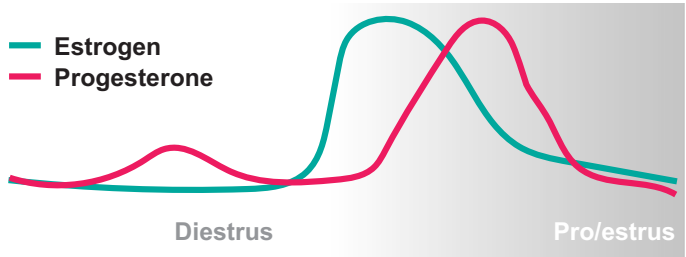

D Cue reinforcement
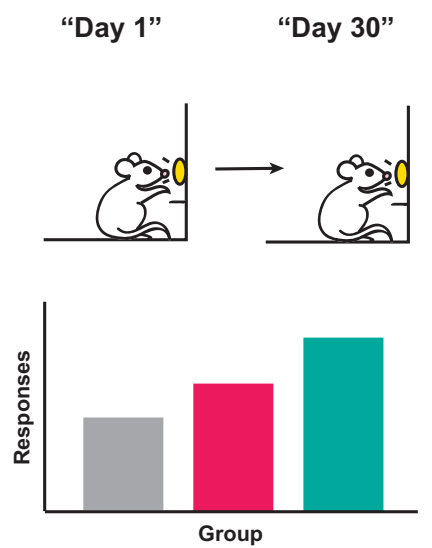

Fig. 1 a Left: Relative levels of circulating estrogen and progesterone across the follicular and luteal phases of the 28-day human menstrual cycle. Right: Relative levels of circulating estrogen and progesterone across the pro/estrus and diestrus phases of the 4-day rodent estrous cycle. b Upper: Initially, males rats and female rats in either pro/estrus or diestrus underwent Pavlovian conditioning to pair i.v. cocaine infusions with the presentation of a cue light ("paired" sessions). Lower: To control for non-specific pairing effects, separate groups of rats also experienced i.v. cocaine infusions in the absence of the cue light ("unpaired" sessions). c Upper: In the threshold or "behavioral economics" procedure, rats self-administered i.v. cocaine under a fixed-ratio 1 schedule of reinforcement across trials with a descending series of cocaine unit doses. As unit doses decreased, the price or number of responses per milligram of cocaine increased. This allowed measurement of the maximal unit price paid or $P_{\max }$ a metric of appetitive or motivated behavior. To assess the incentive motivational value of the cocaineconditioned cues on these measures, a subset of sessions were performed in the presence of the cue (Pavlovian-to-instrumental transfer or "PIT"). Lower: Notably, compared to the "no cue" condition, cues paired with i.v. cocaine during pro/estrus enhanced motivation for cocaine by increasing $P_{\max }$ in females independent of their current cycle status. This potentiating effect was absent for cues conditioned during diestrus and in males. d Upper: In the absence of cocaine, rats lever-pressed for presentations of the conditioned cue on Day 1 and Day 30 after the last cocaine self-administration session. Lower: On Day 30 but not Day 1, females responded more for cocaine cues conditioned during pro/ estrus vs. diestrus or in males

identify neural substrates that may contribute to persistent cueinduced cocaine seeking in females.

Overall, this study by Johnson et al. [5] highlights a fundamental but previously unexplored role played by the estrous cycle in promoting female vulnerability to addiction. Cocaine-paired cues-by virtue of being conditioned during pro/ estrus-gain heightened incentive motivational value that invigorates cocaine intake regardless of current estrous cycle status. Cocaine reward and/or associative learning processes may be enhanced during pro/estrus compared to diestrus, and this is supported by prior work demonstrating estrus-specific increases in brain stimulation reward [7], cocaine conditioned place preference [4], and incubation of cocaine craving [8]. Together, these findings suggest a potential feed-forward mechanism whereby potentiated cocaine reward learning in pro/estrus engenders cocaine-paired cues with augmented incentive properties that serve to promote future and ongoing cocaineseeking behavior. In light of the escalating opioid epidemic and the recent legalization of cannabis, additional investigations will be required to determine if these effects generalize to other classes of abused drugs. These findings, however, undoubtedly add to our understanding of susceptibility to addiction in females and will likely have important implications for both the etiology and treatment of drug abuse.

\section{FUNDING AND DISCLOSURE}

NEZ is supported by NIH/NIDA grant F32 DA043967. The author declare no competing interests.

\section{ACKNOWLEDGEMENTS}

Much appreciation for the insightful comments of Dr. Yavin Shaham and for assistance with figure design from Dr. Jennifer Wenzel.

\section{ADDITIONAL INFORMATION}

Publisher's note: Springer Nature remains neutral with regard to jurisdictional claims in published maps and institutional affiliations.

\section{REFERENCES}

1. Becker JB, Koob GF. Sex differences in animal models: focus on addiction. Pharmacol Rev. 2016;68:242-263.

2. Anker JJ, Carroll ME. Females are more vulnerable to drug abuse than males: evidence from preclinical studies and the role of ovarian hormones. Curr Top Behav Neurosci. 2011;8:73-96.

3. Grimm JW, Hope BT, Wise RA, Shaham Y. Neuroadaptation. Incubation of cocaine craving after withdrawal. Nature. 2001;412:141-142. 
4. Calipari ES, Juarez B, Morel C, Walker DM, Cahill ME, Ribeiro E, et al. Dopaminergic dynamics underlying sex-specific cocaine reward. Nat Commun. 2017;8:13877.

5. Johnson AR, Thibeault KC, Lopez AJ, Peck EG, Sands LP, Sanders CM et al. Cues play a critical role in estrous cycle-dependent enhancement of cocaine reinforcement. Neuropsychopharmacology. 2019; https://doi.org/10.1038/s41386-0190320-0.
6. Carroll ME, Lynch WJ. How to study sex differences in addiction using animal models. Addict Biol. 2016;21:1007-1029.

7. Steiner M, Katz RJ, Carroll BJ. Detailed analysis of estrous-related changes in wheel running and self-stimulation. Physiol Behav. 1982;28:201-204.

8. Nicolas C, Russell TI, Pierce AF, Maldera S, Holley A, You Z-B et al. Incubation of cocaine craving after intermittent access self-administration: sex differences and estrous cycle. Biol Psychiatry. 2019; https://doi.org/10.1016/j.biopsych.2019.01.015. 